Artículo de opinión

\title{
La cardiopatía congénita del adulto: un desafío de salud del presente y del futuro
}

Grown-up congenital heart disease: a present and future challenge

Víctor Robles-Velarde (iD ${ }^{1, a}$

Recibido 15 de agosto de 2020 Aceptado 16 de agosto de 2020

Filiación

a Médico cirujano cardiovascular.

- Servicio de Cirugía Cardiovascular

de Adultos - Instituto Nacional

Cardiovascular INCOR. Lima, Perú.

Correspondencia

Jr Coronel Zegarra 417. Jesús María

Lima.

Correo

victoroblesccv@gmail.com

Conflictos de interés

El autor declara no tener ningún

conflicto de interés.

Financiamiento

Autofinanciado.

Citar como:

Robles Velarde V. La cardiopatía

congénita del adulto: un desafío de

salud del presente y futuro [edito-

rial]. Arch Peru Cardiol Cir Cardiovasc.

2020;1(3):135-138. doi: 10.47487/

apcyccv.v1i3.73.

\section{Introducción}

El estudio y el tratamiento de las cardiopatías congénitas del adulto tiene sus orígenes desde que se advirtió que la supervivencia de los niños nacidos con esta patología aumentaba gracias a los progresos en su diagnóstico y tratamiento. En 1947, Helen B. Taussig publica el libro Congenital Malformation of The Heart iniciando el estudio sistematizado de las cardiopatías congénitas. Años antes, en 1938, Robert E. Gross realiza la primera ligadura del conducto arterioso permeable y, en 1944, Helen B. Taussig y Alfred Blalock efectúan en una niña cianótica con tetralogía de Fallot, la primera fístula sistémico pulmonar logrando la mejoría clínica de la paciente. Luego, en 1953, se utilizó por primera vez la bomba corazón-pulmón de Gibbon para la reparación bajo visión directa de una comunicación interauricular, comenzando así la corrección quirúrgica no solo de los defectos congénitos del corazón, sino de toda la patología cardiovascular.

Por todo lo descrito y ante la aparición de una nueva población de pacientes, en 1984 se funda la International Society for Adult Congenital Heart Disease (ISACHD), una organización sin fines de lucro para promover, mantener y buscar la excelencia en el cuidado de adultos con enfermedad cardíaca congénita en todo el mundo. El año 2001, el reporte de la American Heart Association, estimó que el $85 \%$ de los niños que nacen con una cardiopatía alcanzan la vida adulta y, por primera vez, el número de adultos con cardiopatías congénitas supera al de los niños (1). El 2012, el American Board of Medical Specialties aprobó el establecimiento de una nueva subespecialidad con necesidad de certificación: especialista en Cardiopatías Congénitas del Adulto.

\section{Una población creciente}

El desarrollo de las nuevas técnicas de diagnóstico, de nuevos métodos de intervención y de tratamiento quirúrgico han prolongado la supervivencia de los niños que nacen con una cardiopatía congénita (CC) ${ }^{(1-3)}$, llegando a alcanzar edades que no eran posibles anteriormente. La supervivencia de los pacientes con CC depende de qué tan complejo es el defecto, cuándo se diagnostica y cómo es tratado. Con la mejora del tratamiento de las CC se ha originado una población de adolescentes y adultos que alcanza mejoría de la clase funcional, de la calidad de vida y del pronóstico. Otro grupo de pacientes con CC no reciben ningún tratamiento, o no son diagnosticados en etapas tempranas de la vida, Ilegando a la adultez presentando sintomatología de alteraciones cardiovasculares propias de la historia natural de la enfermedad.

Por lo tanto, los pacientes adultos con CC son aquellos pacientes que fueron sometidos a algún tipo de tratamiento en la infancia como intervencionismo o cirugía y aquellos supervivientes naturales 
de su patología. Muchos de estos pacientes que sobreviven, presentan lesiones residuales, secuelas, complicaciones que pueden evolucionar durante la vida adulta y presentarse como alteraciones eléctricas, enfermedades valvulares, cortocircuitos persistentes, disfunción miocárdica, lesiones vasculares, alteración de los materiales protésicos implantados, infecciones como endocarditis, alteraciones tromboembólicas, alteraciones extracardiacas con afección a diversos órganos y sistemas, alteraciones psicológicas y muerte prematura (Tabla 1) ${ }^{(4,5)}$.

\section{Población afectada}

El informe de la 32. a Conferencia de Bethesda el año 2001, estimó que había alrededor de 2800 adultos con cardiopatías congénitas por cada millón de habitantes, más de la mitad de ellos con defecto de complejidad moderada o alta ${ }^{(6)}$. Se estima que aproximadamente 1 millón de niños y 1,4 millones de adultos estadounidenses viven con una CC y este número está previsto que continúe en ascenso a un ritmo de $5 \%$ por año ${ }^{(1,7)}$. Se estima que el número de adultos con cardiopatías congénitas que viven actualmente en Europa sea de 1 millón de pacientes ${ }^{(8)}$.

Diversos trabajos han establecido que la incidencia de las CC es de aproximadamente $0,8 \%$, con un rango que va de 0,4 a $1,2 \%{ }^{(9-11)}$, las variaciones dependen del momento del estudio, de la población y de los métodos diagnósticos. Dentro de los tipos, las acianóticas son las más frecuentes, representando un $83 \%$ de todas las cardiopatías congénitas, y las cianóticas un 17\% ${ }^{(12)}$. En el Perú tenemos pocos trabajos sobre la incidencia de CC, en el estudio de Olortegui y Adrianzen del año 2007, calcula una incidencia de 8 por 1000 nacidos ${ }^{(12)}$. Según el censo de población del Perú del año $2017^{(13)}$, nacieron alrededor de 600000 niños, entonces aproximadamente nacen en el Perú alrededor de 4000 niños por año con alguna cardiopatía que va a requerir algún tipo de atención médica y muchos de ellos llegarán a la edad adulta con necesidades de atención médica especializada. En el Perú se desconoce el número de pacientes adultos que son portadores

Tabla 1. Clasificación de los residuos, secuelas y complicaciones de las cardiopatías congénitas

\begin{tabular}{|c|c|}
\hline \multirow{2}{*}{ Alteraciones electrofisiológicas } & Cambios electrofisiológicos permanentes \\
\hline & Arritmias y defectos de la conducción \\
\hline \multirow{3}{*}{ Alteraciones valvulares } & Malformaciones intrínsecas de las válvulas \\
\hline & Secuelas de intervenciones anteriores \\
\hline & Efectos hemodinámicos sobre válvulas normales \\
\hline \multirow{2}{*}{ Cortocircuitos persistentes } & Residuos no corregidos \\
\hline & Secuelas de procedimientos terapéuticos \\
\hline \multirow{3}{*}{ Disfunción miocárdica } & Alteraciones estructurales \\
\hline & Hipertrofia y remodelamiento \\
\hline & Isquemia perioperatoria \\
\hline \multirow{3}{*}{ Alteraciones vasculares } & Estenosis congénitas y adquiridas \\
\hline & Hipertensión pulmonar o sistémica \\
\hline & Aneurisma, disección o rotura \\
\hline Alteraciones de materiales protésicos & Parches, válvulas, conductos \\
\hline \multirow{3}{*}{ Complicaciones infecciosas } & Endocarditis \\
\hline & Vasos arteriales o fístulas \\
\hline & Estructuras extravasculares \\
\hline \multirow{2}{*}{ Fenómenos tromboembólicos } & Trombosis intravascular \\
\hline & Tromboembolia pulmonar o sistémica \\
\hline \multirow{6}{*}{ Alteraciones extravasculares } & Desarrollo psíquico y físico \\
\hline & Órganos de los sentidos \\
\hline & Estructura osteomuscular \\
\hline & Sistema nervioso central \\
\hline & Dentición \\
\hline & Otros órganos y sistemas \\
\hline
\end{tabular}

Fuente: Cardiopatías congénitas del adulto: residuos, secuelas y complicaciones de las cardiopatías congénitas operadas en la infancia. José María Oliver Ruiz. Rev Esp Cardiol 2003;56(1):73-88. 


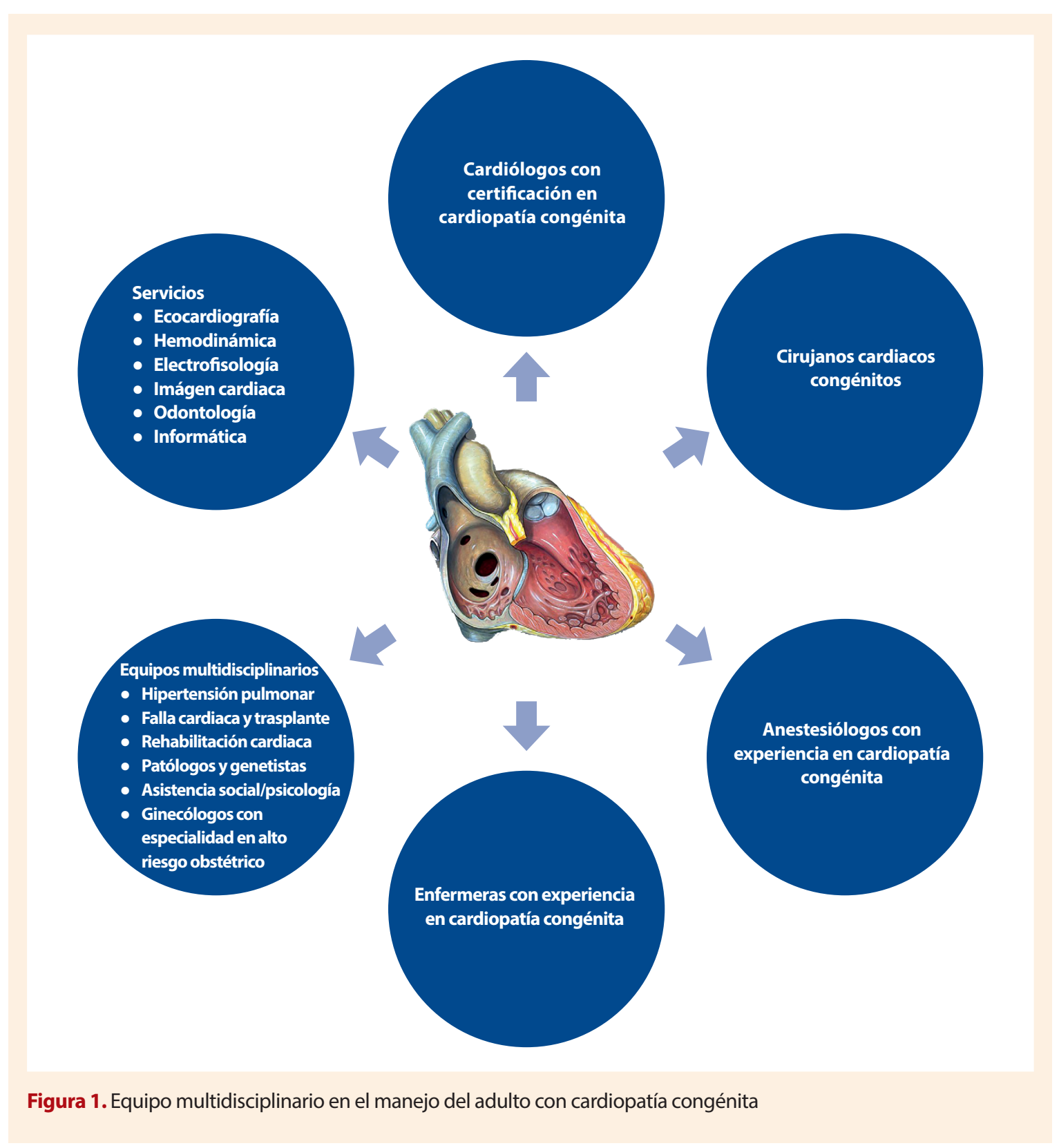

de alguna CC, si nos guiamos por las estimaciones de la 32. ${ }^{\mathrm{a}}$ Conferencia de Bethesda, debe existir casi 90000 adultos portadores de esta patología.

\section{Desafíos en la atención del paciente adulto con cardiopatía congénita}

Con el trascurso de los años tenemos cada vez mayor incremento de pacientes adultos afectados con CC; por lo que hay que considerar: ¿qué necesidades nuevas se están produciendo?, ¿cómo se deben planificar estos nuevos requerimientos?, ¿cómo encontrar soluciones a esta nueva problemática de salud? Para conocer el número de pacientes con cardiopatía congénita que llegará a la edad adulta en los próximos años, se debe conocer primero el número de niños que nacen con una CC en nuestra población; luego, determinar el número de pacientes sometidos a algún tipo de tratamiento y sus niveles de supervivencia. Todo ello permitirá determinar las necesidades de recursos y su distribución en los diferentes niveles de atención de la salud.

La importancia de este grupo poblacional se refleja en la publicación de guías de práctica clínica que se van actualizando periódicamente ${ }^{(14-17)}$, reconociendo los avances en el cuidado de la creciente población de adultos con CC. Hoy en día existen pocas instituciones dedicadas a la atención del adulto con CC; $y$, la enseñanza de esta patología, como parte de la preparación del cardiólogo de adultos, es aún escasa en la mayoría de los centros cardiológicos.

\section{Necesidad de recursos}

Hay una creciente demanda de recursos de salud por parte de este grupo de pacientes, que se traduce en mayor número 
de consultas médicas, de hospitalizaciones, de procedimientos hemodinámicos, de cirugías cardiacas y otros ${ }^{(18,19)}$. Son pacientes que, en ocasiones, han sido sometidos a múltiples cirugías, múltiples intervenciones, hospitalizaciones frecuentes, con afecciones de otros órganos y sistemas; lo que requiere el trabajo conjunto de múltiples especialidades en forma sincronizada (Figura 1).

En concordancia con los desafíos presentes y futuros, en el Instituto Nacional Cardiovascular (INCOR-EsSalud) de Lima-Perú (centro de referencia nacional), se creó el año 2019 el Comité Multidisciplinario Para el Manejo de la Patología Cardiaca Congénita del Adulto; $y$, entre septiembre del 2010 a julio del 2020 se han sometido a tratamiento quirúrgico más de 500 pacientes adultos con CC de diversa complejidad (que incluyen cinco sometidos a trasplante cardiaco), cifra aún insuficiente dada el aumento creciente de esta población.

\section{Conclusiones}

La CC del adulto es una entidad clínica que aumenta continuamente, con nuevas problemáticas y nuevos desafíos. Está presente en el paciente desde antes de nacer y la tendrá hasta el momento en que muera, por lo que su manejo debe involucrar a un equipo multidisciplinario para el manejo óptimo de este grupo especial de pacientes; por ello es importante la creación de centros de referencia especializados.

Ha llegado el momento de preparar al sistema de salud para este grupo creciente de pacientes que requieren atención permanente, integral y altamente especializada. Lamentablemente, en la actualidad no existen suficientes especialistas ni centros de atención que estén capacitados para asumir este reto en nuestro país.

\section{Referencias bibliográficas}

1. Warnes CA, Liberthson R, Danielson GK, et al. Task Force 1: The Changing Profile of Congenital Heart Disease in Adult Life. JACC 2001;37:1161-98. DOI: 10.1016/s0735-1097(01)01272-4.

2. Wren C, O'Sullivan JJ. Survival with congenital heart disease and need for follow up in adult life. Heart. 2001;85:438-43. DOI: doi. org/10.1136\%2Fheart.85.4.438.

3. Boneva RS, Botto LD, Moore CA, et al. Mortality associated with congenital heart defects in the United States. Trends and racial disparities, 1979-1997. Circulation. 2001;103:2376-81. DOI: doi. org/10.1161/CIRCULATIONAHA.110.947002.

4. Engelfriet $P$, Boersma $E$, Oechslin $E$, et al. The spectrum of adult congenital heart disease in Europe: morbidity and mortality in a 5 year follow-up period. European Heart Journal 2005;26:2325-2333. DOI: 10.1093/eurheartj/ehi396.

5. Oliver-Ruiz JM. Cardiopatías congénitas del adulto: residuos, secuelas y complicaciones de las cardiopatías congénitas operadas en la infancia. Rev Esp Cardiol 2003;56(1):73-88. DOI. doi.org/10.1016/ S0300-8932(03)76824-9.

6. Weeb GD, Williams RG. 32nd Bethesda Conference: Care of the adult with congenital heart disease. J Am Coll Cardiol. 2001;37:1161-98. DOI: 10.1016/S0735-1097(01)01279-7.

7. Marelli AJ, lonescu-Ittu R, Mackie AS, et al. Lifetime Prevalence of Congenital Heart Disease in the General Population from 2000 to 2010. Circulation. 2014; 130:749-756. DOI: doi.org/10.1161/ CIRCULATIONAHA.113.008396.

8. Moons $\mathrm{P}$, Bovijn L, BudtsW, et al. Temporal trends in survival to adulthood among patients born with congenital heart disease from 1970 to 1992 in Belgium. Circulation 2010;122:2264-2272. DOI: 10.1161/CIRCULATIONAHA.110.946343.

9. Martínez OP, Romero IC, Alzina de Aguilar V. Incidencia de las cardiopatías congénitas en Navarra (1989-1998). Rev Esp Cardiol. 2005;58(12):1428-34. DOI: 10.1016/S0300-8932(05)74073-2.

10. Hoffman JI, Kaplan S, Liberthson RR. Prevalence of congenital heart disease. Am Heart J. 2004;147(3):425-39. DOI: 10.1016/j. ahj.2003.05.003.
11. Harris JA, Francannet C, Pradat P, et al. The epidemiology of cardiovascular effects, part 2: a study based on data from three large registries of congenital malformations. Pediatr Cardiol. 2003;24(3):222-35. DOI: 10.1007/s00246-002-9402-5.

12. Olortegui A, Adrianzen M. Incidencia estimada de las cardiopatías congénitas. en niños menores de 1 año en el Perú. An Fac Med Lima 2007; 68(2).http://www.scielo.org.pe/scielo.php?script=sci_arttext\&pid=S102555832007000200003\&lng=es.

13. Censos Nacionales 2017: XII de Población, VII de Vivienda y III de Comunidades Indígenas. INEI. Perú. https://www.inei.gob.pe/media/ MenuRecursivo/publicaciones_digitales/Est/Lib1539/libro.pdf.

14. Deanfield J, Thaulow E, Warnes C, et al. Management of Grown Up Congenital Heart Disease. The Task Force on the Management of Grown Up Congenital Heart Disease of the European Society of Cardiology. European Heart Journal 2003;24:1035-1084. DOI: doi. org/10.1016/S0195-668X (03)00131-3.

15. Warnes CA, Williams RG, Bashore TM, et al. ACC/AHA 2008 Guidelines for the Management of Adults with Congenital Heart Disease: A Report of the American College of Cardiology/American Heart Association Task Force on Practice Guidelines. Circulation. 2008;118: e714-e833. DOI: doi.org/10.1161/CIRCULATIONAHA.108.190811.

16. Baumgartner $\mathrm{H}$, Bonhoeffer $\mathrm{P}$, De Groot $\mathrm{N}$, et al. ESC Guidelines for the management of grown-up congenital heart disease (new version 2010). The TaskForce on the Management of Grown-upCongenital Heart Disease of the European Society of Cardiology (ESC). European Heart Journal 2010;31:2915-2957.DOI: doi.org/10.1161/CIRCULATIONAHA.108.190811.

17. Stout KK, Daniels CJ, Aboulhosn JA, et al. 2018 AHA/ACC Guideline for the Management of Adults with Congenital Heart Disease. Circulation. 2019;139: e698-e800. DOI: doi.org/10.1161/CIR.0000000000000603.

18. González CZ. El adulto con cardiopatía congénita. Manejo multidisciplinario de una población creciente. Arch Cardiol Mex 2005; 75: 389-392. http://www.scielo.org.mx/scielo.php?script=sci_ arttext\&pid=S1405-99402005000400002\&lng=es.

19. Sandoval N. Adultos con cardiopatías congénitas: una población creciente. Retos del presente y el futuro. Rev Colomb Cardiol. 2017;24(3):199-203. DOI: 10.1016/j.rccar.2017.05.002 